\title{
Interpretation of Quiescent Behavior of Iron K-Alpha (Fe K $\alpha$ ) Emission Line from Sigma Gem
}

\author{
Ogbodo O.O.V ${ }^{1}$, Chima A.I ${ }^{2 *}$, Onyia A.I ${ }^{3}$ \\ ${ }^{1,2,3}$ Department of Industrial Physics, Enugu State University of Science and Technology \\ Correspondence Author: abraham.chima@esut.edu.ng
}

Available online at: www.isroset.org

Received: 25/Jul/2019, Accepted: 15/Aug/2019, Online: 31/Aug/2019

\begin{abstract}
$\overline{\text { Abstract- In this research the interpretation of Suzaku spectral observation of Fe ka emission line from Sigma Gem system was }}$ carried out. Sigma Gem was observed using Suzaku satellite with observation ID 402033010 at an exposure time of $142.82 \mathrm{ks}$. Spectral analysis of all observations was performed using XSPEC version 12.8. We used version 2.00 of the standard Suzaku pipeline products and the HEA Soft version 6.16 for our analysis of data. We adopted a $250^{\prime \prime}$ radius to extract all events for the XIS detector to produce the source spectra, but we adjusted the $250^{\prime \prime}$ radius slightly where it overlaps with the calibration sources at the corners, to avoid capturing source background light. Modeling the spectrum using either power law or bremsstrahlung model with three Gaussian line for the $6.4 \mathrm{keV}, \mathrm{He}-$ like $6.7 \mathrm{keV}, \mathrm{H}-\mathrm{like} 7.0 \mathrm{keV}$, Fe K $\alpha$ emission lines shows that the $6.4 \mathrm{keV}, 7.0 \mathrm{keV}$ lines and absorption in both full and partial covering matter could not be measured in all the sources. We were able to resolve the three-narrow iron $\mathrm{k} \alpha$ emission lines with different ionization states, which constraints the Sigma Gem emission models. The iron K- line complex was clearly resolved into three individual peaks at $6.41 \mathrm{keV}, 6.7 \mathrm{keV}$ and 7.0 $\mathrm{keV}$. The light curve shows that the Sigma Gem was at a quiescent state at the point of observation and The light curve shows a considerable quiescent behavior of the source of the point of observation showing the source is not flaring.
\end{abstract}

Keywords :Iron k-line, x-ray, sigma gen

\section{INTRODUCTION}

Iron $\mathrm{K}$-alpha $(\mathrm{Fe} \mathrm{K} \alpha)$ emission lines result when an electron transits to the innermost " $\mathrm{K}$ " shell (principal quantum number 1) from a $2 p$ orbital of the second or "L" shell (with principal quantum number 2) of atom releasing $6.4 \mathrm{keV}$ of energy as florescence emission line photon [1]. The line is actually a doublet with slightly different energies depending on spin-orbit interaction between the electron spin and the orbital momentum of the $2 \mathrm{p}$ orbital. $\mathrm{Fe} \mathrm{K} \alpha$ lines are the strongest spectral lines emitted by an element bombarded with energy sufficient to cause maximally intense X-ray emission.

The $\mathrm{Fe} \mathrm{K} \alpha$ emission line is a veritable tool used for the study of the geometry, physics and kinematics of astrophysical sites. As an example, the thermal origin of the $\mathrm{X}$-ray emission from galaxy clusters was resolved with the detection of the $6.7 \mathrm{keV}$ iron line in their X-ray Spectra [2]. At the hottest temperatures iron will become hydrogen-like (Fe XXVI), when it is completely ionized and give rise to a $\mathrm{k} \alpha$ line at $6.97 \mathrm{keV}$. At temperatures of a few $\mathrm{keV}$ iron will be helium -like (Fe XXV), producing a $\mathrm{K} \alpha$ line at $6.70 \mathrm{keV}$
[3]. The cold iron can produce a fluorescent line at 6.41 $\mathrm{keV}$. It was observed firstly using the CCD detectors on the ASCA satellite, which gave $120-\mathrm{eV}$ resolution $[4,5,6]$. An example of $\mathrm{K}$-alpha lines is those seen for iron as iron atoms radiating X-rays spirall into a black hole at the center of a galaxy. For such purpose the energy, $\mathrm{E}$ of the line is adequately calculated to 2-digit accuracy by the use of Moseley's law:

$$
\begin{aligned}
& \mathrm{E}=(10.2 \mathrm{eV})(\mathrm{Z}-1)^{2} \\
& {[1.1]} \\
& \text { where } \mathrm{Z} \text { is the atomic number. }
\end{aligned}
$$

For example, $\mathrm{K}$-alpha for iron $(\mathrm{Z}=26)$ is calculated in this fashion as follows.

$10.2 \mathrm{eV}(25)^{2}=6.38 \mathrm{keV}$ energy.

For astronomical purposes, Doppler and other effects (such as gravitational broadening) show iron line to be better than $6.4 \mathrm{keV}$. From the analysis of the $\mathrm{K} \alpha$ emission spectra, basic physical properties within the emitting region can be deduced, such as temperatures, densities, excitation conditions, ionization balance, elemental abundances and electric - and magnetic field distributions $[7,8]$. 


\section{MATERIALS}

We obtained our data from the High Energy Astrophysics data archive research Centre Japan. SIGMA GEM was observed using Suzaku satellite on 2007-10-21 at 09:48:59pm with observation ID $($ ObsID $)=402033010$ at an exposure time of $142.82 \mathrm{ks}$.

\section{METHOD}

Some high energy astrophysics software was used in analyzing data in this research, these are XSELECT, XSPEC and XIS. We used the XSELECT to extract Suzaku data into spectral, images and light curves as shown in Figures $1-3$

\section{RESULTS}

This section contains the result of the analysis as shown in Table 1 and 2 as well as Table 3 and 4 which gives the estimated data parameters of the source mean quantities from the $\mathrm{Fe} \mathrm{K} \alpha$ line measurements.

Table 1: The Fe lines fit parameters (flare period).

\begin{tabular}{|c|c|c|c|c|c|c|c|c|c|c|}
\hline $\mathrm{kT}$ & PP Index & $\mathrm{F}_{\text {cont }}$ & $\mathrm{E}_{6.7}$ & $\mathrm{E}_{7.0}$ & $\mathrm{~F}_{6.7}$ & $\mathrm{~F}_{7.0}$ & $\mathrm{EW}_{6.7}$ & $\mathrm{EW}_{7.0}$ & $\mathrm{R} \chi^{2}$ & dof \\
\hline- & $3.60 \pm 0.05$ & $4.57 \pm 0.02$ & $6.33 \pm 0.01$ & - & $2.17 \pm 0.03$ & - & $401_{-50}^{+79}$ & - & 1.72 & 44 \\
& & & & & & & & & & \\
\hline
\end{tabular}

Table 2: Bremsstrahlung fit parameters (flare period).

\begin{tabular}{|c|c|c|c|c|c|c|c|c|c|c|}
\hline KT & $\begin{array}{c}\text { PP } \\
\text { Index }\end{array}$ & $\mathrm{F}_{\text {cont }}$ & $\mathrm{E}_{6.7}$ & $\mathrm{E}_{7.0}$ & $\mathrm{~F}_{6.7}$ & $\mathrm{~F}_{7.0}$ & $\mathrm{EW}_{6.7}$ & $\mathrm{EW}_{7.0}$ & $\mathrm{R} \chi^{2}$ & dof \\
\hline $2.54 \pm 0.28$ & - & $0.84 \pm 0.01$ & $6.33 \pm 0.01$ & - & $2.21 \pm 0.32$ & - & $415_{-62}^{+95}$ & - & 1.75 & 44 \\
\hline
\end{tabular}

Table 3: The Fe lines fit parameters (quiescence period).

\begin{tabular}{|c|c|c|c|c|c|c|c|c|c|c|}
\hline Kt & PP Index & $\mathrm{F}_{\text {cont }}$ & $\mathrm{E}_{6.7}$ & $\mathrm{E}_{7.0}$ & $\mathrm{~F}_{6.7}$ & $\mathrm{~F}_{7.0}$ & $\mathrm{EW}_{6.7}$ & $\mathrm{EW}_{7.0}$ & $\mathrm{R} \chi^{2}$ & dof \\
\hline- & $3.99 \pm 0.18$ & $2.90 \pm 0.18$ & $6.64 \pm 0.01$ & - & $1.52 \pm 0.03$ & - & $479_{-50}^{+99}$ & - & 0.56 & 79 \\
\hline
\end{tabular}

Table 4. Bremsstrahlung fit parameters (quiescence period).

\begin{tabular}{|c|c|c|c|c|c|c|c|c|c|c|}
\hline $\mathrm{Kt}$ & $\begin{array}{c}\mathrm{PP} \\
\text { Index }\end{array}$ & $\mathrm{F}_{\text {cont }}$ & $\mathrm{E}_{6.7}$ & $\mathrm{E}_{7.0}$ & $\mathrm{~F}_{6.7}$ & $\mathrm{~F}_{7.0}$ & $\mathrm{EW}_{6.7}$ & $\mathrm{EW}_{7.0}$ & $\mathrm{R} \chi^{2}$ & dof \\
\hline $2.11 \pm 0.20$ & - & $1.98 \pm 0.14$ & $6.33 \pm 0.01$ & - & $1.53 \pm 0.16$ & - & $500_{-50}^{+79}$ & - & 0.55 & 79 \\
\hline
\end{tabular}

Parameters in Tables 1,2 \& 3, 4 are: The bremsstrahlung continuum temperature $\mathrm{kT}$ in $\mathrm{keV}$, power law photon Index (PP Index) is dimensionless, the continuum flux in $10^{-2}$ photons $\mathrm{keV}^{-1} \mathrm{~s}^{-1} \mathrm{~cm}^{-2}$ (Fcont), the centroid energies of 6.7, and 7.0 lines in $\mathrm{keV},\left(\mathrm{E}_{6.7}\right.$, and $\left.\mathrm{E}_{7.0}\right)$, the line fluxes in $10^{-5}$ photons $\mathrm{s}^{-1} \mathrm{~cm}^{-2}\left(\mathrm{~F}_{6.7}\right.$, and $\left.\mathrm{F}_{7.0}\right)$, and the equivalent widths in $\mathrm{eV}\left(\mathrm{EW}_{6.7}, \mathrm{EW}_{7.0}\right), \mathrm{R} \chi^{2}$, and dof is the degrees of freedom.
The Figure below is the diagram of the observed light curve of the source Sigma Gem. We argue that ionization equilibrium plasma is likely to have given rise to the observed $\mathrm{He}$-like and $\mathrm{H}_{2}-$ like $\mathrm{K} \alpha$ lines. 


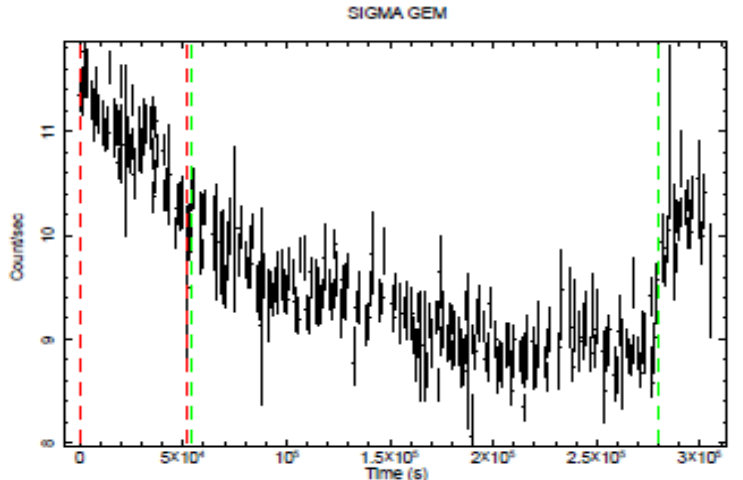

Figure 1: Light Curve of SIGMA GEM showing quiescent behaviour

The two-vertical broken red line enclosed the region of extraction of the stellar flares while the green lines enclosed the region of extraction of the quiescence. Because the light curve shows quiescent behavior, the source is not flickering.

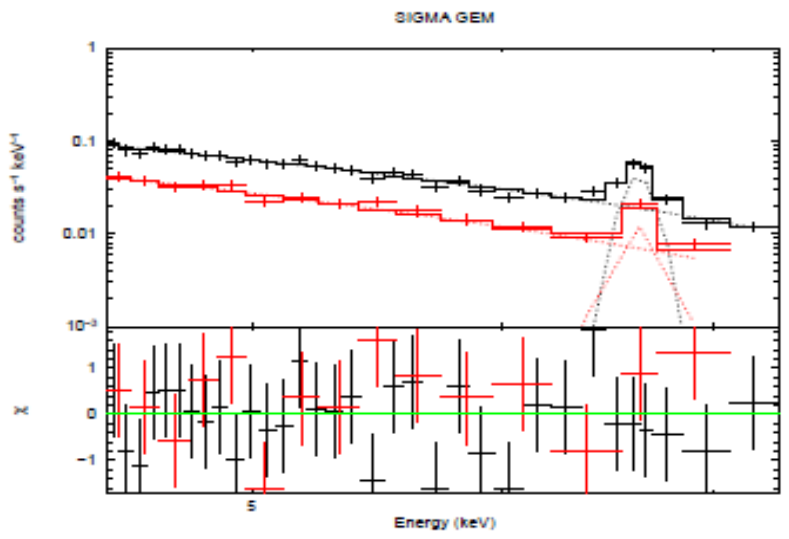

Figure 2:Spectrum of the extracted flares region of SIGMA GEM

In the upper panel, the data and the best-fit model are shown by crosses and solid lines, respectively. The spectral component is represented by dotted lines. In the lower panel, the ratio of the data to the best-fit model is shown by crosses.

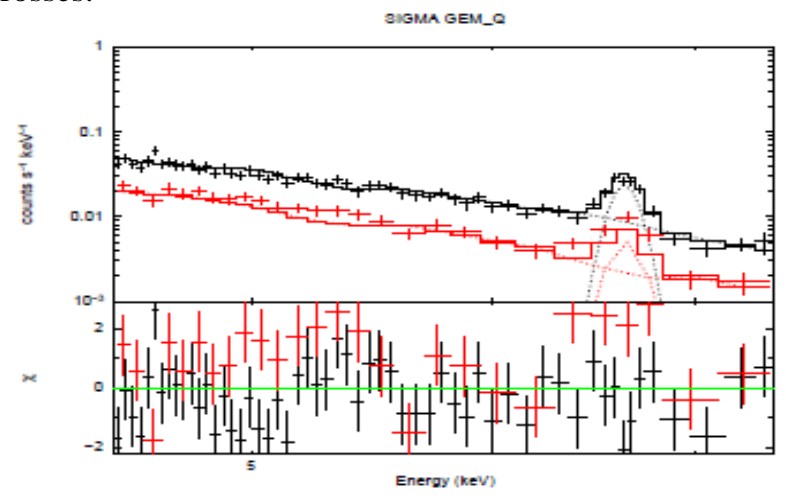

Figure 3: Spectrum of the extracted quiescence region of SIGMA GEM.
In the upper panel, the data and the best-fit model are shown by crosses and solid lines, respectively. The spectral component is represented by dotted lines. In the lower panel, the ratio of the data to the best-fit model is shown by crosses. Hence a simple thermal bremsstrahlung model fit the data.

\section{DISCUSSION}

From the result of the $\mathrm{Fe}$ line fit parameter in the flare period it was observed that the continuum temperature (KT) was not found. The power law photon index is $3.60 \pm 0.05$ with the photon count $\left(F_{\text {cont }}\right)$ of $4.5 \pm 0.02$. For the energy at $6.7 \mathrm{keV}$ it is $6.33 \pm 0.01$. The energy at $7.0 \mathrm{keV}$ was not found, the photon count at $6.7 \mathrm{keV}$ flux is $2.17 \pm 0.03$ while the photon count of $7.0 \mathrm{keV}$ flux was not found. The equivalent width of $6.7 \mathrm{keV}$ is $401_{-50}^{+79}$, the equivalent width at $7.0 \mathrm{keV}$ was not found. The $\mathrm{R} \chi^{2}$ is 1.72. Hence, the model produced statistically unacceptable spectral fit within this X-ray regime.

For the bremsstrahlung fit parameter in the flare period it shows that the continuum temperature (KT) is $54 \pm 0.28$, the power law photon index was not found while the photon count $\left(\mathrm{F}_{\text {count }}\right)$ is $0.8 \pm 0.01$. The energy at $7.0 \mathrm{keV}$ was not found, the photon count of $6.7 \mathrm{keV}$ flux is $2.21 \pm 0.32$, the photon count of 7.0 flux was not found. The equivalent width at $6.7 \mathrm{keV}$ is $415_{-62}^{+95}$, the equivalent width of $7.0 \mathrm{keV}$ was not found. The $\mathrm{R} \chi^{2}$ value is 1.75 shows that it does not fit the model.

For the Fe lines fit parameters in the quiescence period Table 1. shows that the continuum temperature (KT) was not found, The power law index is $3.99 \pm 0.18$, the photon count $\left(\mathrm{F}_{\text {count }}\right)$ is $2.90 \pm 0.18$. The energy at $6.7 \mathrm{keV}$ is $6.64 \pm 0.01$. The energy at $7.0 \mathrm{keV}$ was not found. While the photon count at $6.7 \mathrm{keV}$ flux is $1.53 \pm 0.16$, the photon count at $7.0 \mathrm{keV}$ flux was not found, the equivalent width at 6.7 $\mathrm{keV}$ is $479_{-50}^{+99}$. The equivalent width at $7.0 \mathrm{keV}$ was not found, the $\mathrm{R} \chi^{2}$ is 0.56 . and hence it fits the model.

For the Bremsstrahlung fit parameter in the quiescence period, the continuum temperature is given as $2.11 \pm 0.20$, the power law photon index was not found with the photon count of about 1.98. The energy at $6.7 \mathrm{keV}$ is $6.33 \pm 0.001$ while the energy at $7.0 \mathrm{keV}$ was not found, the photon count of $6.7 \mathrm{keV}$ flux is $1.53 \pm 0.16$, while the photon count of $7.0 \mathrm{keV}$ flux was not found. The equivalent width of 6.7 $\mathrm{keV}$ is $500_{-50}^{+79}$, the equivalent width for $7.0 \mathrm{KeV}$ was not found, the $\mathrm{R} \chi^{2}$ is 0.55 ; hence it fits the model. The Fe line fit parameter and the bremsstrahlung fit parameter both in flaring and in quiescence period all fit the model because their $\mathrm{R} \chi^{2}$ values lies below 1 . 
The Fe line fit parameter and bremsstrahlung fit parameter in the flare period does not fit the model because the $\mathrm{R} \chi^{2}$ values are above 1 while the quiescence period of the $\mathrm{Fe}$ line fit parameter and bremsstrahlung fit parameter fits the model because their $\mathrm{R} \chi^{2}$ values is above 1 .

We therefore confirm that the hard X-ray from these our source originates from the shock front close to the surface of the white dwarf magnetic pole due to bremsstrahlung cooling by electrons. Other important observation is the presence of strong $\mathrm{H}$-like and He-like emission line detected at line centroid energy of 6.4, 6.7 and $7.0 \mathrm{keV}$. (and a detached in the Suzaku XIS spectra of Sigma Gem).

Another significant aspect of the result is the light curve of Sigma Gem generated using the XIS combined data after background subtraction. Although detailed timing analysis of the combined spectrum, which could enable in-depth discussion of all the possible variability in the source is not yet available, the light curve of the Sigma Gem shows no detachable flaring activity at the time of observation. This suggest that no significant changes in some observable physical process, such as increase in the acceleration rate, occurred in the system at the time it was observed.

\section{SUMMARY}

Spectral analysis of all observations was performed using XSPEC version 12.8. We used version 2.00 of the standard Suzaku pipeline products and the HEA Soft version 6.16 for our analysis of data. We adopted a $250^{\prime \prime}$ radius to extract all events for the XIS detector to produce the source spectra, but we adjusted the $250^{\prime \prime}$ radius slightly where it overlaps with the calibration sources at the corners, to avoid capturing source background light. The XIS background spectra were extracted with $250^{\prime \prime}$ radius with no apparent sources and were offset from both the source and corner calibrations. We also adjusted the radius accordingly in some cases where it overlaps with the calibration sources at the corners. The light-curve was generated and we extracted only the portion where the source showed stellar flares (see the mark region in the source light curve shown in Fig. 1). Response Matrices File and Ancillary Response File were generated for the XIS detector for the extracted stellar flares region using the FTOOLS which is in line with [9], respectively. In our models we assumed two types of absorption by fullcovering and partial covering matter. Since we were primarily interested in the ion lines, our fitting covers $4.5-$ $7.5 \mathrm{keV}$ for the XIS BI and the XIS FI. Modeling the spectrum using either power law or bremsstrahlung model with three Gaussian line for the $6.4 \mathrm{keV}$, He-like $6.7 \mathrm{keV}$, $\mathrm{H}$-like $7.0 \mathrm{keV}, \mathrm{Fe} \mathrm{K} \alpha$ emission lines shows that the 6.4 $\mathrm{keV}, 7.0 \mathrm{keV}$ lines and absorption in both full and partial covering matter could not be measured in all the sources. Statistically acceptable fit could only be obtained if we model with power law or bremsstrahlung with a Gaussian line for the $6.7 \mathrm{keV}$ line. The $6.4 \mathrm{keV}$ and $7.0 \mathrm{keV}$ emission lines are completely absent. Our inability to measure the absorption in both full and partial covering could be attributed to low photon count/s in the extracted stellar flares spectra. We also extracted the quiescence period spectra in these stars (see Fig.2) and repeated our analysis procedure as in the flares region. We also found that there are no significant differences between the EWs and bremsstrahlung continuum temperatures of the flares and quiescence periods of the star (see Tables 1,2,3 and 4). Spectra of all the sources were presented in Figure 1,2 and 3 and spectral parameters were shown in Tables $1,2,3$ and 4.

The Fe $\mathrm{K} \alpha$ line can be used to deduce the values of some physical parameters such as temperatures, densities, excitation conditions, ionization balance, elemental abundances and electric and magnetic field distributions in high energy astrophysical sites as suggested by previous authors $[10,11,12]$

We found that the physical processes going on in high energy astrophysical sites such as mCVs, hard X-ray emitting symbiotic stars, $\mathrm{X}$-ray binaries and AGN is mainly from the $\mathrm{X}$-ray spectra, which has the $\mathrm{Fe} \mathrm{K} \alpha$ line as a main feature. The iron abundance in these sources are far more than any other heavy metal, hence the iron fluorescent emission line is a hallmark of the accretion driven X-ray sources [13].

We were able to also use the Fe $\mathrm{K} \alpha$ emission line to get the better understanding of the geometry, physics and kinematics of the high energy astrophysical sites. We discovered that the state ionization in a system can also be deduced by the line centroid energy.

\section{CONCLUSION}

The iron K-line complex was clearly resolved into three individual peaks but only $6.41 \mathrm{keV}$ and $6.7 \mathrm{keV}$ were deduced in this research. The major findings are:

The light curve shows a considerable quiescent behavior of the source of the point of observation showing the source is not flaring. The $6.4 \mathrm{keV}$ is produced at the shock front due to collusional ionization of flaring gas between the inner accretion disk and the surface of white dwarf. The $6.7 \mathrm{keV}$ is produced by the reflection of surface by the magnetized white dwarf.

\section{REFERENCES}

[1] Aizu, K. (1973). X-Ray Emission Region of a White Dwarf with Accretion Theoretical physics, journal letter vol. 49, no. 1184.

[2] Mitchell, R. J, Culhane, J. L Davison, P. J., \& Ivesm J. C., (1976). Monthly Notices of the Radio Astronomy Society, 176, 29. 
[3] Makishimak, (1986). in Mason K. O., Watson M. G., White N. E. eds the physics of accretion onto compact objects. SpringerVerlag, Berlin, p.249.

[4] Hellier, C., Mason, K. O., Rosen, S. R., \& Cordova, F. A. (1989). Accretion-powered Compact BinariesMonthly Notices of the Radio Astronomy Society, 228, 463.

[5] Helliler, C., (2001). Cataclysmic variable stars-how and why they vary, Springer Science and Business media, New York.

[6] Heintz, W. D. (1978). Double Stars. Dordrecht: D. Reidel Publishing Company. pp. 1-2.

[7] Jacobs, V. L., Decaux, V., \& BeiersdorFer, P., (2004). X-ray Diagnostics of Astrophysical plasmas Cambridge, MA, United States.

[8] Kahn, S. M., Behar, E., Kinkhabwala, A., \&Savin D. W., (2002). the Royal Society, 360, 1923.

[9] Boldt, E. (1987). Astronomy and astrophysics with the Advanced X-ray Astrophysics Facility. Physics Report 146:216.

[10] Eze, R. N. C, (2013). Fe K Alpha Line in Hard X- ray emitting symbiotic star Monthly Notices of the Royal Astronomical Society.

[11] Esaenwi, S., Eze, R.N.C., (2014). New Astronomy 2, Sol-gel synthesis, optical and structural characterization of $\mathrm{ZrOS}$ nanopowder.

[12]Matranga, M.; Drake, J.J.; Kashyap, V.L.; Marengo, M. Kuchner, M. J. (2010). Close Binaries with Infrared Excess: Destroyers of Worlds, The Astrophysical Journal Letters, Volume 720, Issue 2, L164-L168.

[13] Piro, L., (1993). Iron K Line Diagnostics in Astrophysical Sources Press) adsabs Harvard edu 71993 yxrs. Oof. 4484.

\section{AUTHORS PROFILE}

Mrs Ogbodo Osondu Oluchukwu Vivian have Bachelor of Science and Master of Science from Department of Industrial Physics, Enugu State University of Science and Technology. Currently she is a $\mathrm{PhD}$ student of the same Department.

Chima Iheanyichukwu Abraham have Bachelor of Science, Master of Science and Doctor of Philosophy from Department of Industrial Physics, Enugu State University of Science and Technology. $\mathrm{He}$ is a member of Astronomical Society of Nigeria and a member of Nigerian Institute of Physics. He has published over 24 journals in highly reputed International Journals. He has 5 years of teaching experience and 2 years of Research experience.

Onyia Ike Augustin have Bachelor of Science, and Master of Science from Department of Physics and Astronomy University of Nigeria Nsukka and Doctor of Philosophy from Department of Industrial Physics, Ebonyi State University Abakaliki . He is a member of Nigerian Institute of Physics. He has published more than 27 journals in highly reputed international journals. He has 35 years of teaching experience and 20 years of Research experience. 